\title{
Estudio comparativo de los métodos para evaluar la transmitancia térmica en cerramientos opacos en el invierno mediterráneo
}

\section{A comparative study of the methods to assess the thermal transmittance in opaque walls in the Mediterranean winter}

\author{
D. Bienvenido-Huertas ${ }^{(*)}$, R. Rodríguez-Álvaro ${ }^{(*)}$, J. Moyano $^{(*)}, \underline{\text { D. Marín }}{ }^{(*)}$, F. Rico ${ }^{(*)}$
}

\section{RESUMEN}

En España, la mayor parte del parque inmobiliario presenta un comportamiento energético deficiente. Para paliar dicha situación, se debe analizar, entre otros aspectos, su envolvente. De las diferentes propiedades que caracterizan los cerramientos, la transmitancia térmica es una de las más significativas. Sin embargo, las metodologías de evaluación in situ están planteadas para climas fríos, y los métodos teóricos tienen asociados una incertidumbre elevada. Ante esta circunstancia, resulta necesario estudiar su aplicación en climas suaves como el mediterráneo. El presente estudio analizó tanto los métodos teóricos existentes como los experimentales. Para ello se realizó una monitorización sobre cuatro casos de estudio durante el invierno. Los resultados pusieron de manifiesto que, con unas condiciones metrológicas correctas y una estabilidad de las condiciones ambientales, se pueden obtener resultados representativos.

Palabras clave: Transmitancia térmica, UNE-EN ISO 6946, ISO 9869-1, método termografía infrarroja cuantitativa, mediterráneo, invierno.

\section{ABSTRACT}

In Spain, most of the building stock has an inefficient energy performance. To change this situation, among other aspects, building envelopes must be analysed to change this situation. Thermal transmittance is one of the most significant properties related to building envelopes. However, in-situ evaluation methodologies for this property are designed for cold climates. On the other hand, theoretical methods have a high associated uncertainty. Therefore, it is necessary to study the application of these in situ methods in mild climates such as the Mediterranean one. This study analyses both theoretical and experimental methods. For this purpose, four case studies have been monitored during the winter. Results show that representative results could be obtained with adequate metrological conditions and stable environmental conditions.

Keywords: Thermal transmittance, UNE-EN ISO 6946, ISO 9869-1, quantitative infrared thermography method, Mediterranean, winter.

(*) Escuela Técnica Superior de Ingeniería de Edificación, Universidad de Sevilla. Sevilla (España).

(**) Escuela Técnica Superior de Ingenieros de Caminos, Canales y Puertos, Universidade da Coruña. La Coruña (España). Persona de contacto/Corresponding author: damar@us.es (D. Marín).

ORCID: https://orcid.org/oooo-0003-0716-8589 (D. Bienvenido-Huertas); https://orcid.org/oooo-0002-5920-9496 (R. Rodríguez-Álvaro); https://orcid.org/oooo-0oo2-2186-6159 (J. Moyano); https://orcid.org/oooo-0002-7652-5730 (D. Marín); https://orcid.org/oooo-0002-2390-8949 (F. Rico).

Cómo citar este artículo/Citation: Bienvenido-Huertas, D.; Rodríguez-Álvaro, R.; Moyano, J.; Marín, D.; Rico, F. (2019). Estudio comparativo de los métodos para evaluar la transmitancia térmica en cerramientos opacos en el invierno mediterráneo. Informes de la Construcción, 71(554): e288. https://doi.org/10.3989/ic.62542

Copyright: (c) 2019 CSIC. Este es un artículo de acceso abierto distribuido bajo los términos de la licencia de uso y distribución Creative Commons Reconocimiento 4.0 Internacional (CC BY 4.0). 


\section{INTRODUCCIÓN}

La creciente preocupación por el cambio climático ha hecho que la Unión Europea, a través del Desafío Social del Horizonte2020 "Energía Segura, Limpia y Eficiente", haya establecido como uno de los objetivos prioritarios para la comunidad científica la reducción del consumo de energía en el sector de la construcción.

La transmitancia térmica (U) constituye uno de los parámetros fundamentales de la envolvente del edificio que afecta a su demanda energética, por lo que la determinación correcta de su valor es fundamental para el establecimiento de Medidas de Ahorro Energético (MAEs) eficientes, con unos períodos de amortización cortos.

Li et al. (1) y Stevens y Bradford (2) determinaron a través de sus investigaciones que la mayor parte de los inmuebles que analizaron tienen una demanda energética sobredimensionada, fruto de una mala estimación de la transmitancia térmica de sus cerramientos. Esto supuso para los dueños de dichas viviendas un incremento innecesario en la inversión económica con el fin de mejorar el comportamiento energético del edificio.

Por tanto, los diferentes métodos de evaluación de transmitancia térmica constituyen una herramienta con un gran potencial en la propuesta de mejoras energéticas de los edificios existentes. Tal importancia queda reflejada ante el gran número de investigaciones existentes en los últimos años acerca de los diferentes métodos de determinación, tanto teóricos como experimentales.

La obtención de un valor erróneo de transmitancia térmica de un cerramiento influye en el cálculo de otros aspectos relacionados con la actuación a realizar (el rango de horas de confort y la demanda energética, entre otros), con lo que se puede desembocar en la propuesta de medidas poco adaptadas a la realidad con un incremento en los períodos de amortización económica.

Así pues, determinar de manera correcta esta variable de los edificios resulta fundamental para reducir el potencial consumo energético y las emisiones de $\mathrm{CO} 2$ en un parque inmobiliario con unos valores de aislamiento térmico deficientes (3).

Sin embargo, los métodos de ensayo in situ establecen unas condiciones ambientales demasiado restrictivas, puesto que exigen una gran diferencia de temperatura entre el ambiente exterior y el interior para obtener valores precisos. Este requisito presenta una discordancia con el clima propio de Andalucía (España), caracterizado por temperaturas cálidas a lo largo de todo el año. Algunas ciudades de esta comunidad, como por ejemplo Sevilla, llegan a presentar temperaturas medias muy superiores a las del resto de ciudades ubicadas en la misma tipología climática (Csa-Mediterránea según el sistema de Köppen (4)). Aunque en una primera instancia se puede pensar que el mayor rango de disconfort térmico en esta región tiene lugar en las épocas estivales, Sánchez-García et al. (5) concluyeron que el mayor porcentaje de horas fuera de los límites de confort térmico adaptativo tiene lugar en invierno.

Ante esta circunstancia, el objetivo del presente estudio consiste en analizar la viabilidad de aplicar los métodos teóricos y experimentales en cuatro casos de estudio durante la es- tación de invierno en la ciudad de Sevilla, ya que es en este período cuando se pueden dar unas condiciones ambientales más propicias para realizar los ensayos.

\section{LOS MÉTODOS DE TRANSMITANCIA TÉRMICA}

Los métodos que se evalúan en este trabajo son:

- Método de cálculo teórico (UNE-EN ISO 6946) (6).

- Método termoflujométrico (ISO 9869-1) (7).

- Método cuantitativo mediante termografía infrarroja: Albatici y Tonelli (8),(9).

- Método cuantitativo mediante termografía infrarroja: Dall'O et al. (10).

\subsection{Cálculo teórico (UNE-EN ISO 6946)}

Uno de los métodos más extendidos para el cálculo de la transmitancia térmica es el método de cálculo teórico basado en la norma UNE-EN ISO 6946.

Este método se basa en el cálculo de la transmitancia térmica estacionaria de un cerramiento convencional a través del espesor y de la conductividad térmica de sus diferentes capas, así como de sus resistencias térmicas superficiales.

Por lo tanto, la transmitancia térmica se obtiene mediante la siguiente formulación:

$$
\begin{gathered}
R_{j}=\frac{d_{j}}{\lambda_{j}} \\
R_{T}=R_{s i}+\sum_{j=1}^{n} R_{i}+R_{s e} \\
U_{6946}=\frac{1}{R_{T}}
\end{gathered}
$$

Donde:

- $d_{j}$ y $\lambda_{j}$ son el espesor $[\mathrm{m}]$ y la conductividad térmica $[\mathrm{W} /$ $(\mathrm{m} \cdot \mathrm{K})]$ de cada capa del cerramiento, y $R_{j}$ es la resistencia térmica obtenida para dicho elemento $\left[\mathrm{m}^{2} \cdot \mathrm{K} / \mathrm{W}\right]$.

- $R_{s i}$ y $R_{s e}$ son las resistencias térmicas superficiales interior y exterior del cerramiento $\left[\mathrm{m}^{2} \cdot \mathrm{K} / \mathrm{W}\right]$, y $R_{T}$ es la resistencia térmica total del cerramiento $\left[\mathrm{m}^{2} \cdot \mathrm{K} / \mathrm{W}\right]$.

- $R_{6946}$ es la transmitancia térmica $\left[\mathrm{W} /\left(\mathrm{m}^{2} \cdot \mathrm{K}\right)\right]$ obtenida según la norma UNE-EN ISO 6946.

Los datos relativos al espesor de las capas del cerramiento pueden obtenerse de varias maneras: consultando documentación técnica, realizando una estimación a partir de la medida del espesor total del cerramiento y del conocimiento de su fecha de construcción, o midiendo directamente sobre un testigo extraído del cerramiento. Este último procedimiento suele ser más costoso y molesto para el usuario del edificio (11), aunque permite determinar con exactitud su composición. En cuanto a los valores de conductividad térmica, estos pueden obtenerse a través de diferentes bases de datos como la norma UNE-EN ISO 10456 (12), el Catálogo de Elementos Constructivos (13) o los datos facilitados por el fabricante de los materiales. Por último, es necesario disponer de un valor de resistencia térmica superficial interior y exterior mediante la utilización de los valores predefinidos en la UNE-EN ISO 6946 (6). 
A pesar de su amplia utilización, el valor obtenido por este método tiene un alto nivel de incertidumbre. Como ya se ha comentado, un porcentaje de la desviación asociada puede disminuirse mediante la extracción de testigos (11). Si bien esta compensación va asociada al espesor de los materiales, no tiene en cuenta el error generado por la utilización de diferentes valores de conductividad térmica.

Otro factor que influye en la incertidumbre del resultado son las variaciones que presentará la conductividad térmica en función de la temperatura y humedad ambientales (14),(15). En este sentido, la mayoría de las bases de datos establecen unas condiciones ambientales fijas para las propiedades térmicas de los materiales. Sin embargo, estos valores pueden variar según la latitud y longitud del lugar en el que se encuentre el edificio, por lo que habrá una gran diferencia entre el comportamiento térmico esperado y el real (16). Ante esta situación, Pérez-Bella et al. (17) establecieron unos coeficientes de ponderación para las diferentes capitales de provincia en España que permiten aumentar la precisión del método, aunque su utilización está limitada a seis tipologías de cerramiento opaco.

$$
R_{\text {tcorregido }}=R_{s i}+\sum_{j=1}^{n} \frac{d_{j}}{C C F n_{j}}+R_{\text {se }}
$$

Donde $C C F$ es el coeficiente de ponderación para cada capital española propuesto por Pérez-Bella et al. (17).

\subsection{Análisis termoflujométrico (ISO 9869-1)}

El análisis termoflujométrico (HFM) es un método de evaluación de transmitancia térmica que parte de la medición periódica de las temperaturas interior y exterior del edificio y del flujo de calor que atraviesa el cerramiento a analizar. Las primeras se miden mediante sondas de temperatura, mientras que la medición del flujo se realiza con un termoflujómetro. Se trata de un método bastante explorado desde el punto de vista científico y está regulado por la norma ISO 9869-1 (7). Los datos de temperatura y flujo de calor pueden tratarse de distintas maneras para obtener el resultado de la transmitancia térmica. El método de la media asimila que el régimen de flujo es estacionario y requiere de diferencias de temperatura elevadas entre el interior y el exterior del edificio. $\mathrm{Al}$ revisar la literatura científica, se determina que para poder obtener unos resultados fiables, la diferencia de temperatura entre los ambientes exterior e interior debe ser mayor de $10{ }^{\circ} \mathrm{C}(11),(18),(19)$ o de $15{ }^{\circ} \mathrm{C}(8)$. $\mathrm{Al}$ conseguir esta gran diferencia de temperaturas, se permite despreciar el efecto de la capacidad térmica de las diferentes capas o materiales del elemento de estudio. Además, el ensayo debe prolongarse durante un mínimo de $72 \mathrm{~h}$ y hasta que el valor de transmitancia térmica resultante se haya estabilizado (7), aunque existen estudios que utilizan períodos temporales mayores (18),(20). Según este método, el valor de transmitancia térmica se obtiene de la siguiente expresión:

$$
U_{9869-1}=\frac{\sum_{j=1}^{n} q_{j}}{\sum_{j=1}^{n}\left(T_{i j}-T_{e j}\right)}
$$

Donde:

- $q_{j}$ es el flujo de calor $\left[\mathrm{W} / \mathrm{m}^{2}\right]$ que atraviesa el cerramiento en un instante $j$, y $T_{i j}$ y $T_{e j}$ son las temperaturas ambientales $[\mathrm{K}]$ en el instante $j$ interior y exterior, respectivamente.
Debido a las exigencias de gradiente de temperaturas, existen estudios que pretenden analizar la viabilidad del método en diferentes regiones. Entre estos estudios se puede destacar, por un lado, el realizado por Lorencio Pérez et al. (21) en el que se aplicó la ISO 9869-1 en cerramientos convencionales en clima seco y, por otro lado, el de Domingo de Miguel (22) que estudió su empleo sobre cerramientos de madera en climas oceánicos. Asimismo, Desogus et al. (11) realizó una campaña experimental mediante dos salas climatizadas que simulaban temperaturas propias de los climas mediterráneos, aunque el ensayo no estaba sujeto a la variabilidad de presentar oscilaciones térmicas y a la acción de otras variables (por ejemplo, el viento).

Una forma de validar el resultado obtenido es mediante la utilización del procedimiento incluido en la ISO 9869-1 (7) según el cual se debe obtener el valor de transmitancia térmica de la UNE-EN ISO 6946 (6). Si la diferencia entre ambos valores es inferior al $20 \%$, se puede considerar que el valor obtenido por el método termoflujométrico es representativo (7).

\subsection{Análisis cuantitativo mediante termografía infrarroja}

Ante la dificultad que presenta el método termoflujométrico al necesitar monitorizar una parte concreta del cerramiento en períodos largos de tiempo, algunos autores han desarrollado métodos de obtención con termografía infrarroja (ITT) (8),(10) que tienen como ventajas la brevedad con la que se llevan a cabo las mediciones y la posibilidad de realizar de manera simultánea una valoración cualitativa del cerramiento.

Los métodos recientemente publicados y con un comportamiento similar (23) son los de Albatici y Tonelli (8),(9) y Dall'O et al. (10).

El método de Albatici y Tonelli se fundamenta en la relación entre la potencia térmica transportada a través del cerramiento y el gradiente de temperaturas entre el interior y el exterior. Al descartar las contribuciones por conducción, el método utiliza la ley de Stefan-Boltzman para la contribución por radiación y la ecuación de Jurges (24) para la contribución por convección, obteniendo la siguiente expresión:

$$
U_{A \& T}=\frac{\sigma \varepsilon\left(T_{s e}^{4}-T_{e}^{4}\right)+3,8054 v\left(T_{s e}-T_{e}\right)}{T_{i}-T_{e}}
$$

Donde:

- $\sigma$ es la constante de Stefan-Boltzman $\left[5,67 \cdot 10^{-8} \mathrm{~W} /\left(\mathrm{m}^{2} \cdot \mathrm{K}^{4}\right)\right]$.

- $\varepsilon$ es la emisividad del cerramiento [adimensional].

- $v$ es la velocidad del viento local $[\mathrm{m} / \mathrm{s}]$.

- $T_{s e}$ es la temperatura superficial exterior del muro $[\mathrm{K}]$ y $T_{i}$ y $T_{e}$ son las temperaturas ambientales interior y exterior, respectivamente [K].

Por otro lado, Dall'O et al. (10) propusieron el cálculo de la transmitancia bajo la suposición de que el flujo de calor entre los ambientes separados por una pared se puede expresar como la relación del flujo de calor calculado en función del coeficiente de convección exterior y la diferencia entre la temperatura de la superficie exterior y la del aire exterior. La relación de este flujo de calor con la diferencia entre las tem- 
peraturas del aire interior y exterior da lugar a la siguiente formulación:

$$
U_{\text {Dallo }}=\frac{(5.8+3,8054 v)\left(T_{s e}-T_{e}\right)}{T_{i}-T_{e}}
$$

Donde las variables $T_{s e}, T_{i}, T_{e} \mathrm{y} v$ son las mismas variables que para la ecuación [6].

En la literatura científica se han estudiado de manera amplia ambos métodos, pero siempre bajo unas condiciones ambientales óptimas para su aplicación. A su vez, el método propuesto por Albatici y Tonelli ha sido estudiado en profundidad por los propios autores (8),(9), pero la región donde se realizan los ensayos es el norte de Italia, con unas temperaturas medias más bajas que en otras regiones mediterráneas situadas más al sur. Dall'O et al. (10) validaron su método analizando catorce tipologías de cerramiento diferentes ubicadas en el municipio de Carugate (Milán), con la misma tipología de clima que los casos de estudio de Albatici y Tonelli. Por otro lado, Nardi et al. (23) utilizaron ambos métodos bajo unas condiciones ambientales controladas a través de una caja caliente.

\section{REVISIÓN DE ESTUDIOS COMPARATIVOS}

Existen investigaciones que comparan los distintos métodos de evaluación de transmitancia térmica. En la Figura 1 se recogen de forma gráfica los resultados obtenidos en las diferentes investigaciones analizadas y las desviaciones existentes entre el valor teórico y el medido en los casos de estudio mediante la utilización del criterio de validación del resultado indicado anteriormente para la ISO 9869-1.

Los estudios comparativos o análisis de los métodos de transmitancia térmica que aparecen en la Figura 1 son los siguientes:

- 1: Media de una muestra de 93 cerramientos macizos de ladrillo o piedra, sin aislamiento ni cámara de aire, mediante análisis termoflujométrico (1). Valor teórico correspondiente con la normativa del Chartered Institute of Building Ser-

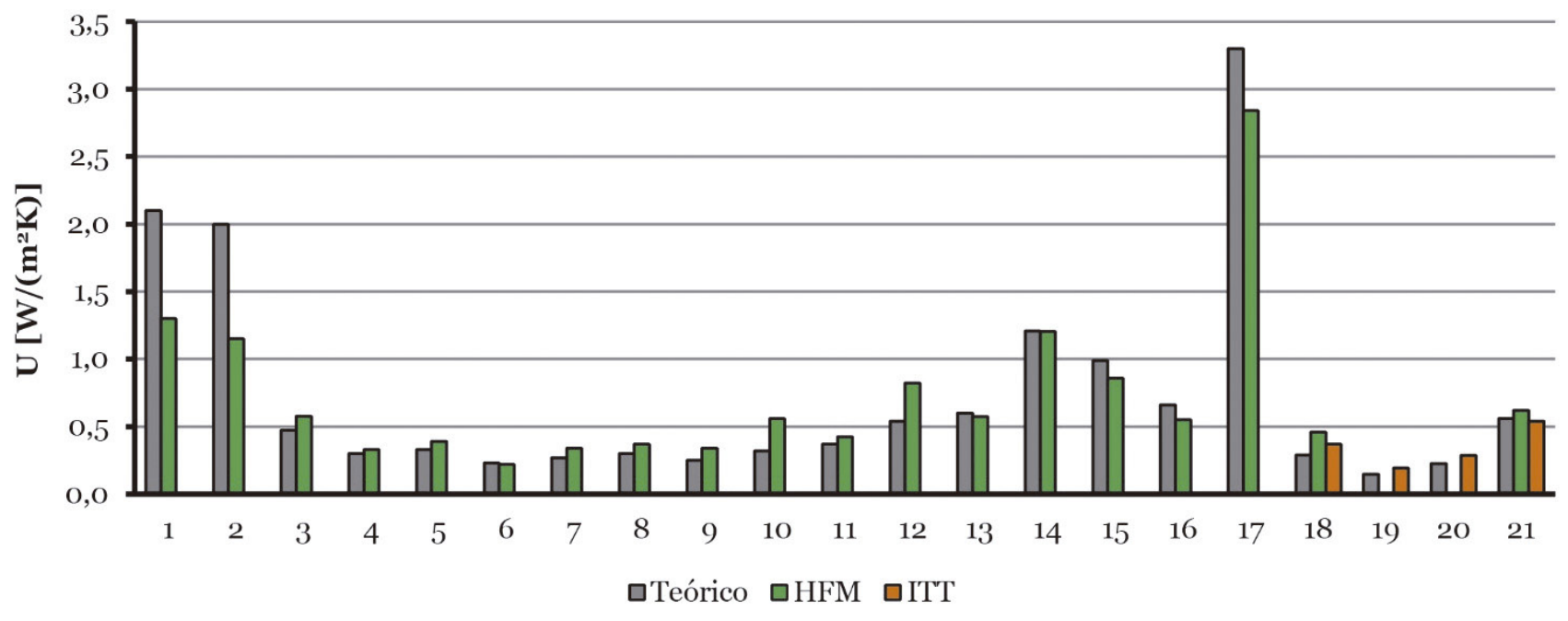

(a)

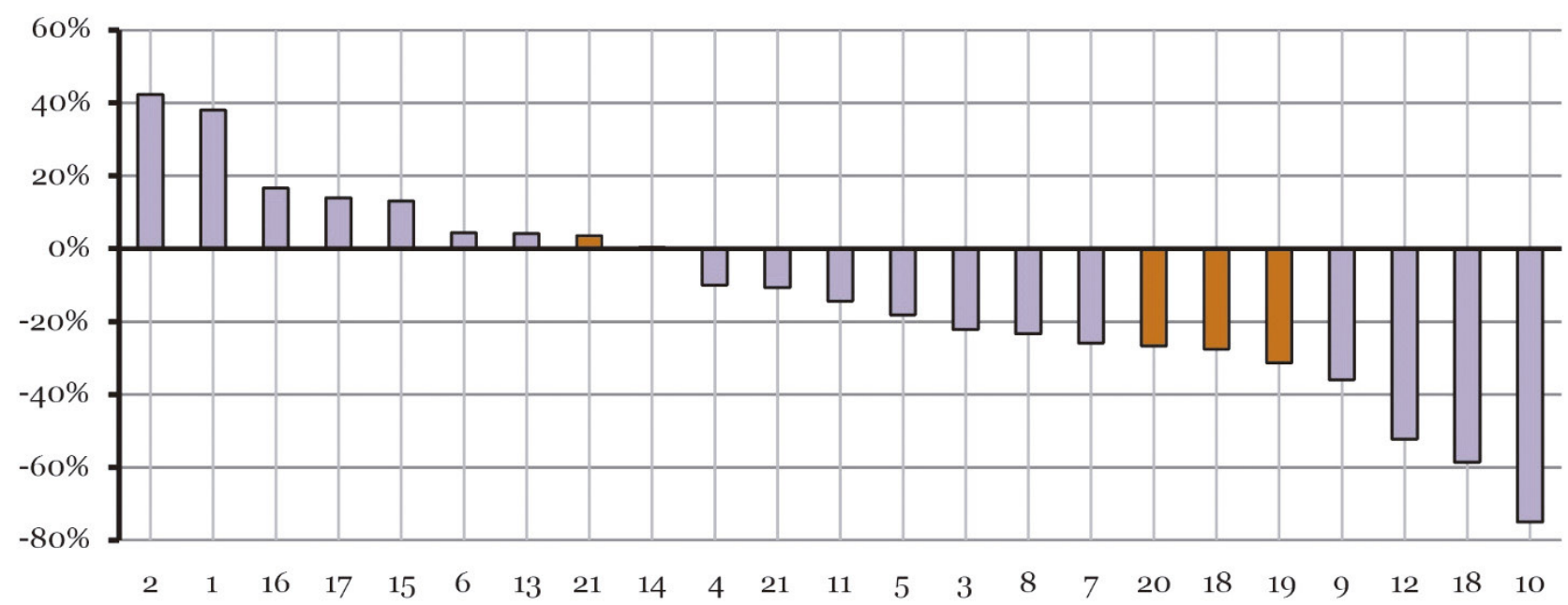

(b)

Figura 1. Revisión de estudios comparativos: (a) resultados de transmitancia térmica obtenidos por diversos autores mediante la utilización de los métodos experimentales de análisis termoflujométrico (HFM) y análisis cuantitativo mediante termografía infrarroja (ITT), comparándolos con los que se obtienen mediante el cálculo teórico; (b) variación del valor de transmitancia térmica obtenido mediante análisis termoflujométrico (violeta) o análisis cuantitativo mediante termografía infrarroja (naranja) respecto del valor obtenido mediante cálculo teórico. 
vices Engineers (CIBSE) y con los procedimientos de certificación energética de edificios existentes de Reino Unido.

- 2: Media de una muestra de 93 cerramientos macizos de ladrillo o piedra, sin aislamiento ni cámara de aire, utilizando el método termoflujométrico (25). El valor teórico se calcula con la conductividad media entre los valores máximo y mínimo que ofrecen varias fuentes consultadas por el autor.

- 3: Media de tres localizaciones distintas sobre un mismo cerramiento convencional de doble hoja con aislamiento intermedio y cámara de aire. Análisis termoflujométrico mediante método de la media (21).

- 4: Análisis termoflujométrico manteniendo una diferencia de temperatura constante de $10^{\circ} \mathrm{C}$ para un cerramiento de hoja simple de ladrillo revestido por ambas caras (11).

- 5-10: Análisis termoflujométrico de los siguientes casos de estudio pertenecientes a edificios con diseños altamente eficientes (19):

- 5: Elemento macizo con aislamiento intermedio.

- 6-9: Cerramiento de doble hoja con aislamiento intermedio y cámara de aire.

- 10: Fachada de un único material opaco sin aislamiento.

- 11-17: Estudio comparativo entre el cálculo teórico con valores medios de transmitancia térmica a partir de una distribución rectangular y los valores obtenidos por endoscopia, y el análisis termoflujométrico de los siguientes cerramientos (18):

- 11: Cerramiento macizo de mampostería con aislamiento por el exterior.

- 12: Panel sándwich de aislamiento recubierto con aluminio.

- 13: Fachada de doble hoja con aislamiento intermedio y cámara de aire.

- 14-16: Elemento macizo de doble hoja sin aislamiento.

- 17: Ventana con vidrio doble y cámara de aire.

- 18-20: Análisis a partir de datos obtenidos mediante termografía infrarroja, utilizando el método establecido por los autores para los siguientes casos de estudio (8):

- 18-19: Envolvente ligera de madera.

- 20: Cerramiento de doble hoja con aislamiento intermedio y sin cámara de aire.

- 21: Cerramiento de una hoja con aislamiento por el exterior sometido a ciclos de diferencia de temperaturas en una caja caliente. Se aplica el análisis termoflujométrico y el análisis cuantitativo mediante termografía infrarroja. Los resultados finales son la media de los obtenidos en cada ciclo (23).

En general, el método teórico es menos preciso a la hora de evaluar la transmitancia térmica de cerramientos macizos, sin aislamiento ni cámara de aire (1),(25), mientras que para cerramientos de tipo convencional es más exacto. Ante este grado de imprecisión, resulta recomendable la extracción de testigos para tener al menos una mayor certeza acerca de la composición constructiva del cerramiento a analizar (18). En general, se puede extraer de la figura que el cálculo teórico arroja con mayor frecuencia valores de transmitancia térmica superiores a los estimados mediante mediciones in situ, en los casos en que el valor de U es elevado $(U>0,5)$. Para valores inferiores suele ocurrir lo contrario. Estas variaciones en ambos sentidos pueden ser debidas, entre otras razones, a que los valores de conductividad térmica de los materiales ofrecidos por los fabricantes sean demasiado bajos por razones de marketing (19) o a que en realidad la conductividad de los materiales es mayor a causa de su contenido en humedad y su grado de envejecimiento (11),(18).

Al margen de estos aspectos, se constata que existen pocos estudios comparativos entre los diferentes métodos. La mayoría de ellos son estudios entre el método termoflujométrico y el teórico, tales como los realizados por los equipos de investigación de Asdrubali et al. (19), Desogus et al. (11) o Ficco et al. (18), aunque en los últimos años también se han realizado estudios entre los métodos termoflujométrico y cuantitativos mediante termografía infrarroja como el realizado por Nardi et al. (23). Sin embargo, no se detecta que exista en la actualidad algún estudio entre todos ellos y en unas condiciones climáticas como las del sur de España.

\section{METODOLOGÍA}

\subsection{Casos de estudio}

Para realizar el estudio comparativo de los diferentes métodos, se seleccionaron cuatro cerramientos situados en la ciudad de Sevilla y libres de radiación solar directa (Tablas 1-4). Se eligió una muestra representativa de las fachadas del parque inmobiliario existente: dos cerramientos que no disponían de aislamiento $\left(\mathrm{C}_{2}\right.$ y $\left.\mathrm{C}_{3}\right)$ y otros dos que sí lo tuvieran (C1 y C4). Otro aspecto considerado a la hora de seleccionar los cerramientos fue que estos pertenecieran a la tipología constructiva típica de la región de Sevilla, caracterizada por la utilización de doble hoja de ladrillo con cámara de aire.

Tabla 1. Composición del cerramiento $\mathrm{C} 1$.

\begin{tabular}{|l|l|c|c|}
\cline { 2 - 4 } \multicolumn{1}{c|}{} & \multicolumn{1}{c|}{ Material } & $\begin{array}{c}\mathbf{d} \\
{[\mathbf{c m}]}\end{array}$ & $\begin{array}{c}\lambda \\
{[\mathbf{W} /(\mathbf{m} \cdot \mathbf{K})]}\end{array}$ \\
\hline 1 & $\begin{array}{l}\text { Mortero de cemento o cal para } \\
\text { revoco }\left(\rho=1900 \mathrm{~kg} / \mathrm{m}^{3}\right)\end{array}$ & 1,5 & 1,3 \\
\hline 2 & Ladrillo perforado & 11,5 & 0,35 \\
\hline 3 & $\begin{array}{l}\text { Mortero de cemento o cal para } \\
\text { revoco }\left(\rho=1900 \mathrm{~kg} / \mathrm{m}^{3}\right)\end{array}$ & 1,0 & 1,3 \\
\hline 4 & Poliestireno extruido (valor medio) & 3,5 & 0,036 \\
\hline 5 & Cámara de aire & 1,0 & - \\
\hline 6 & Ladrillo hueco & 5,0 & 0,32 \\
\hline 7 & $\begin{array}{l}\text { Enlucido de yeso } \\
\left(1000 \mathrm{~kg} / \mathrm{m}^{3}<\rho<1300\right)\end{array}$ & 1,5 & 0,57 \\
\hline
\end{tabular}

Tabla 2. Composición del cerramiento C2.

\begin{tabular}{|l|l|c|c|}
\cline { 2 - 4 } \multicolumn{1}{c|}{} & \multicolumn{1}{c|}{ Material } & $\begin{array}{c}\mathbf{d} \\
{[\mathbf{c m}]}\end{array}$ & $\begin{array}{c}\boldsymbol{\lambda} \\
{[\mathbf{W} /(\mathbf{m} \cdot \mathbf{K})]}\end{array}$ \\
\hline 1 & $\begin{array}{l}\text { Mortero de cemento o cal para } \\
\text { revoco }\left(\rho=1900 \mathrm{~kg} / \mathrm{m}^{3}\right)\end{array}$ & 1,5 & 1,3 \\
\hline 2 & Ladrillo macizo & 11,5 & 0,85 \\
\hline 3 & $\begin{array}{l}\text { Mortero de cemento o cal para } \\
\text { revoco }\left(\rho=1900 \mathrm{~kg} / \mathrm{m}^{3}\right)\end{array}$ & 1,0 & 1,3 \\
\hline 4 & Cámara de aire & 10,5 & - \\
\hline 5 & Ladrillo hueco & 5,0 & 0,32 \\
\hline 6 & $\begin{array}{l}\text { Mortero de cemento o cal para } \\
\text { revoco }\left(\rho=1900 \mathrm{mg} / \mathrm{m}^{3}\right)\end{array}$ & 1,5 & 1,3 \\
\hline 7 & Azulejo cerámico & 1,0 & 1,3 \\
\hline
\end{tabular}

Tabla 3. Composición del cerramiento $\mathrm{C}_{3}$.

\begin{tabular}{|l|l|c|c|}
\cline { 2 - 4 } \multicolumn{1}{c|}{} & \multicolumn{1}{|c|}{ Material } & $\begin{array}{c}\mathbf{d} \\
{[\mathbf{c m}]}\end{array}$ & $\begin{array}{c}\boldsymbol{\lambda} \\
{[\mathbf{W} / \mathbf{( m \cdot K ) ]}}\end{array}$ \\
\hline 1 & $\begin{array}{l}\text { Mortero de cemento o cal para } \\
\text { revoco }\left(\rho=1900 \mathrm{~kg} / \mathrm{m}^{3}\right)\end{array}$ & 1,5 & 1,30 \\
\hline 2 & Ladrillo perforado & 11,5 & 0,35 \\
\hline 3 & $\begin{array}{l}\text { Mortero de cemento o cal para } \\
\text { revoco }\left(\rho=1900 \mathrm{~kg} / \mathrm{m}^{3}\right)\end{array}$ & 1,0 & 1,3 \\
\hline 4 & Cámara de aire & 1,5 & - \\
\hline 5 & Ladrillo hueco & 5,0 & 0,32 \\
\hline 6 & $\begin{array}{l}\text { Enlucido de yeso } \\
\left(1000 \mathrm{~kg} / \mathrm{m}^{3}<\rho<1300\right)\end{array}$ & 1,5 & 0,57 \\
\hline
\end{tabular}


Tabla 4. Composición del cerramiento C4.

\begin{tabular}{|l|l|c|c|}
\cline { 2 - 4 } \multicolumn{1}{l|}{} & \multicolumn{1}{c|}{ Material } & $\begin{array}{c}\mathbf{d} \\
{[\mathbf{c m}]}\end{array}$ & $\begin{array}{c}\lambda \\
{[\mathbf{W} /(\mathbf{m} \cdot \mathbf{K})]}\end{array}$ \\
\hline 1 & Ladrillo perforado & 11,5 & 0,35 \\
\hline 2 & $\begin{array}{l}\text { Mortero de cemento o cal para } \\
\text { revoco }\left(\rho=1900 \mathrm{~kg} / \mathrm{m}^{3}\right)\end{array}$ & 1,5 & 1,3 \\
\hline 3 & Poliestireno extruido (valor medio) & 3 & 0,036 \\
\hline 4 & Cámara de aire & 7 & - \\
\hline 5 & Ladrillo hueco & 7,0 & 0,32 \\
\hline 6 & $\begin{array}{l}\text { Enlucido de yeso } \\
\left(1000 \mathrm{~kg} / \mathrm{m}^{3}<\rho<1300\right)\end{array}$ & 1,5 & 0,57 \\
\hline
\end{tabular}

La configuración constructiva de los cerramientos $\mathrm{C} 2$ y C3 se estimó a partir de su espesor total y del año en el que fueron construidos, ya que se construyeron en el período anterior a la NBE-CT-79 (26), el cual se caracterizó por no utilizar aislamiento en sus soluciones constructivas. Así pues, al caracterizarse la forma de construcción típica de la zona por la ejecución de una doble hoja de ladrillo (una de ladrillo perforado y otra de ladrillo hueco), el espesor del cerramiento variará en función de la anchura que corresponda a la cámara de aire. De esta manera, la estimación con base en el espesor del cerramiento queda justificada para estos dos casos de estudio.

La determinación de $\mathrm{C} 1$ y $\mathrm{C} 4$, correspondientes al período constructivo entre la NBE-CT-79 y el Código Técnico de la Edificación (CTE) (27), se realizó mediante la consulta en documentación técnica fiable. Por su parte, se tomaron los valores medios ofrecidos por el Catálogo de Elementos Constructivos del CTE (13) como valores de conductividad térmica.

La contribución de la cámara de aire a la transmitancia térmica se cuantifica directamente con su valor de resistencia térmica. Según la norma UNE-EN ISO 6946 (6), a los cerramientos 1, 2, 3 y 4 les correspondían valores de resistencia térmica de $0,17,0,15,0,18$ y $0,18\left[\left(\mathrm{~m}^{2} \cdot \mathrm{K}\right) / \mathrm{W}\right]$, respectivamente.

Para la obtención de los valores teóricos se aplicó también el coeficiente corrector propuesto por Pérez-Bella et al. (17) de valor $3,85 \%$ para la ciudad de Sevilla.

\subsection{Monitorización y tratamiento previo de datos}

Para poder aplicar los diferentes métodos descritos anteriormente, se monitorizaron los cuatro cerramientos objeto de estudio utilizando la relación de equipos que aparecen en la Tabla 5. La colocación de los mismos se realizó conforme a lo indicado por las publicaciones existentes de los diferentes métodos (7),(8).(9),(10),(18).

Cada uno de los cerramientos se monitorizó durante un período de tiempo determinado (Tabla 6). Para ello, se midieron registros cada $1 \mathrm{~s}$ para cada una de las variables necesarias de los diferentes métodos. Para establecer los períodos de medición, se procuró que la predicción meteorológica (consultada en la Agencia Estatal de Meteorología) pronosticase siempre ausencia de viento y precipitaciones, así como temperaturas mínimas especialmente frías. De esta manera, se buscaba registrar un mayor gradiente térmico a ambos lados de los cerramientos estudiados al tiempo que se pretendía evitar la influencia de otros fenómenos meteorológicos como el fuerte viento y las precipitaciones sobre el flujo de calor del cerramiento o sobre las propias sondas exteriores
Tabla 5. Especificaciones técnicas del instrumental utilizado

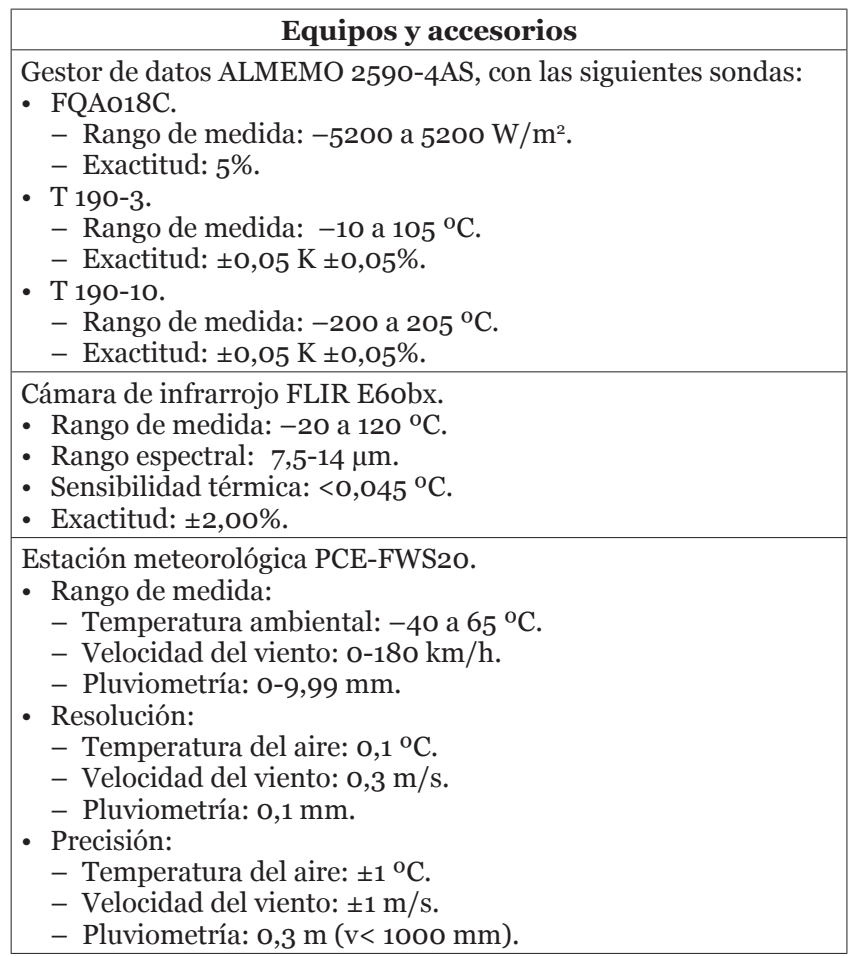

Tabla 6. Períodos de monitorización.

\begin{tabular}{|c|c|c|c|}
\cline { 2 - 4 } \multicolumn{1}{c|}{} & $\begin{array}{c}\text { Duración } \\
\text { (horas) }\end{array}$ & $\begin{array}{c}\text { Frecuencia de } \\
\text { muestreo (s) }\end{array}$ & $\begin{array}{c}\text { Número } \\
\text { de datos }\end{array}$ \\
\hline $\mathrm{C} 1$ & 78,0 & 1 & 4.680 \\
\hline $\mathrm{C} 2$ & 90,5 & 1 & 5.430 \\
\hline $\mathrm{C} 3$ & 117,5 & 1 & 7.050 \\
\hline $\mathrm{C} 4$ & 120,0 & 1 & 7.200 \\
\hline
\end{tabular}

de medición. En este aspecto, el buen tiempo del invierno en Sevilla permitió garantizar unas condiciones adecuadas sin lluvia y con unas velocidades de viento bajas en torno a los cerramientos.

La duración total de los períodos de ensayo se estableció conforme a la norma ISO 9869-1 (7), con una duración de los mismos siempre superior a $72 \mathrm{~h}$ (Tabla 6). Los ensayos finalizaron contando con las preferencias y la disponibilidad de los usuarios de las viviendas.

Para los resultados obtenidos a través de los diferentes métodos, se determinó la incertidumbre estándar combinada utilizando los valores de precisión de los equipos utilizados (Tabla 5). Respecto a los métodos teóricos, se consideró una incertidumbre relativa del $10 \%$ para el valor obtenido a través de la UNE-EN ISO 6946 (6) y de un 5\% para el de la ponderación de PérezBella et al. (17) para las contribuciones debidas al espesor y conductividad térmica estimadas para cada una de las capas.

En cuanto a los métodos cuantitativos de termografía infrarroja, se supuso una emisividad de 0,90 en los cuatro casos de estudio. Este valor es una buena aproximación para las tipologías de cerramiento típicas en la zona (28).

Además, para contar con una mayor información de las condiciones ambientales y poder corroborar los valores medidos, se decidió registrar los datos meteorológicos de la estación de AEMET en Tablada, próxima al lugar de realización de los ensayos. 
Finalmente, para determinar los valores de transmitancia térmica se realizó un post-procesado de los datos obtenidos, utilizando aquellos registros que presentaban un gradiente térmico superior a $7^{\circ} \mathrm{C}$. En este sentido, la utilización de filtrados de datos con un gradiente térmico entre 5 y $10^{\circ} \mathrm{C}$ pueden suponer una posibilidad para obtener resultados válidos en condiciones climáticas desfavorables. La determinación del valor de transmitancia térmica para el método termoflujométrico se realizó mediante la aplicación de la ecuación [5], mientras que para las ecuaciones [6] y [7] se realizó la media aritmética.

\section{RESULTADOS Y DISCUSIÓN}

\subsection{Variables medidas}

En las Figuras 2 y 3 se representan gráficamente las variables medidas para cada uno de los métodos de ensayo.

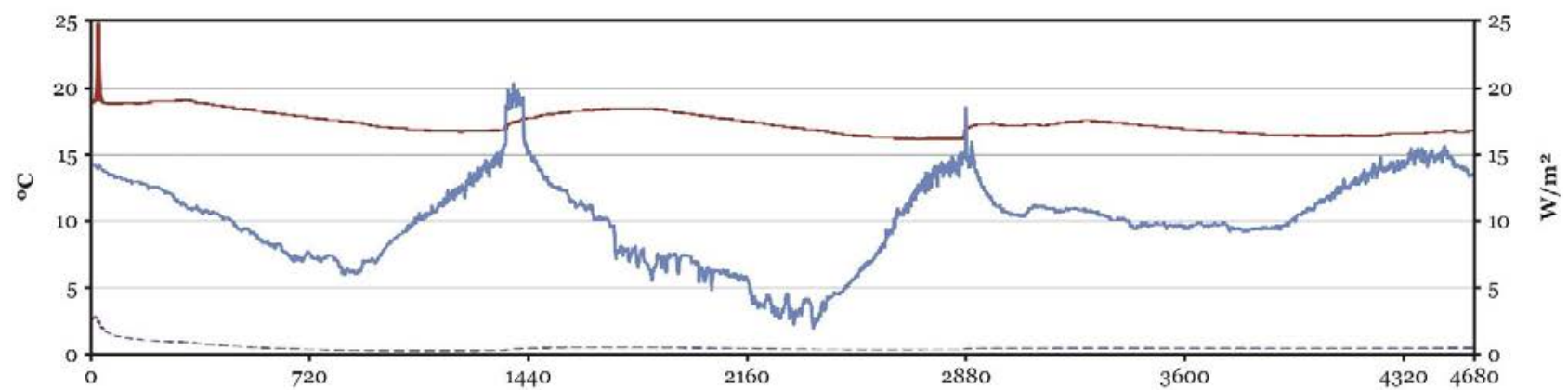

(a)

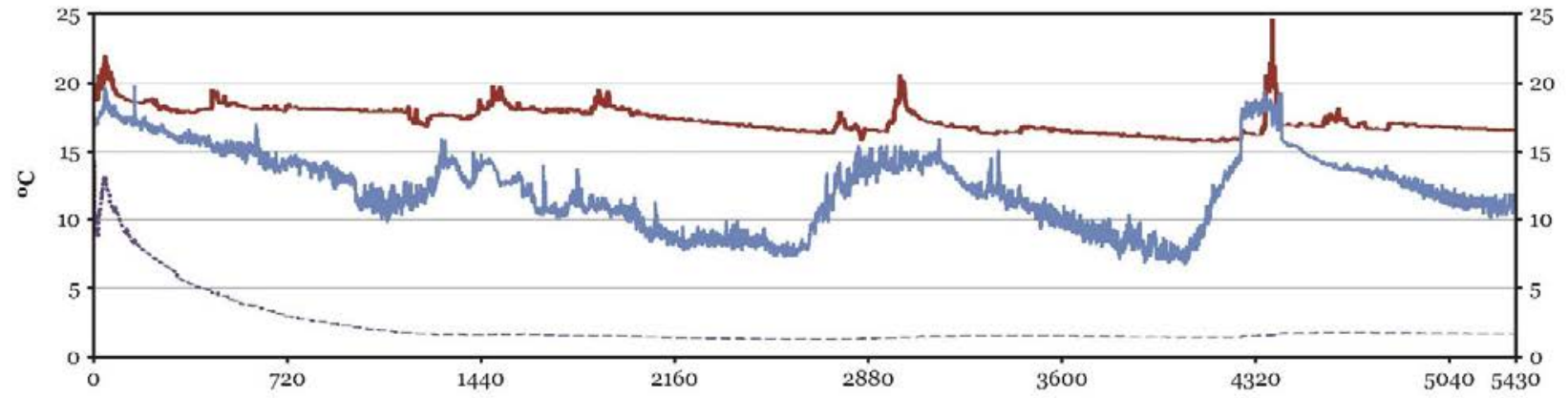

(b)

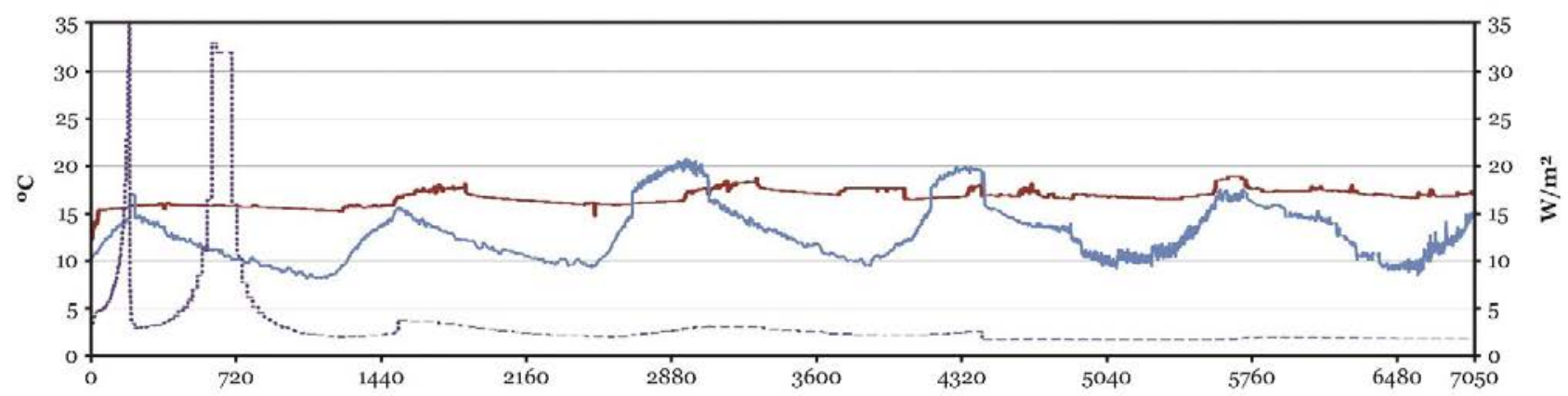

(c)

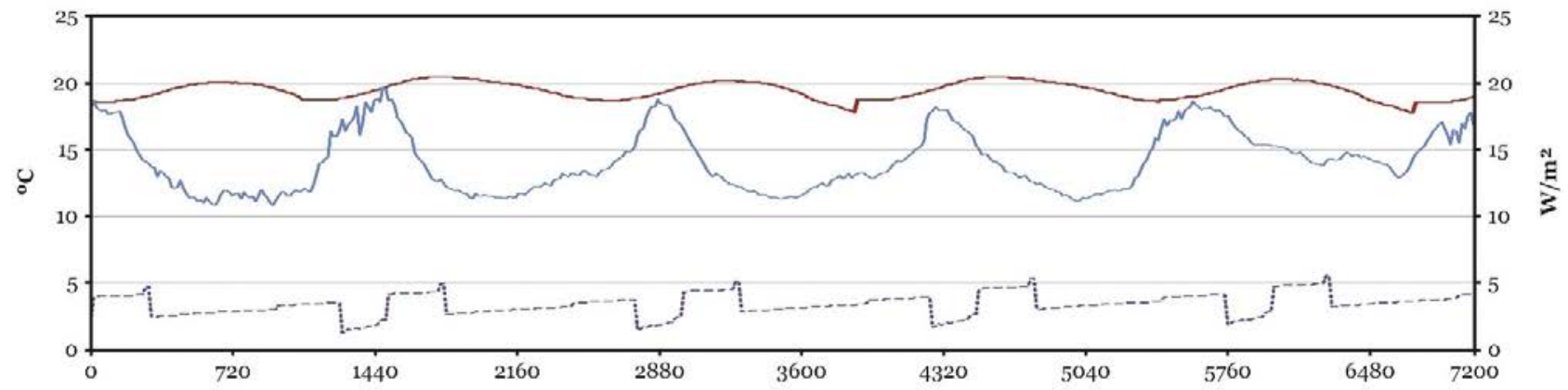

(d) 
$\mathrm{Al}$ analizar los valores registrados, la anomalía más importante que se pudo distinguir fue la caída de los valores de densidad de flujo de calor para los casos C1, C2 y C3 (Figura 2). Los autores consideraron que la causa más probable fue la no interposición de una sustancia de elevada conductividad térmica y con cierta adhesividad entre la placa y el cerramiento. Ante este problema, se decidió interponer un material de estas características en el caso $\mathrm{C}_{4}$. La gráfica referente a este caso muestra cómo efectivamente esta medida surtió efecto, ya que se registraron valores de flujo mayores.

También se puede apreciar cómo la diferencia de temperaturas entre el interior y exterior fue inferior a $10{ }^{\circ} \mathrm{C}$ para casi todos los períodos de estudio, salvo en uno de los días del

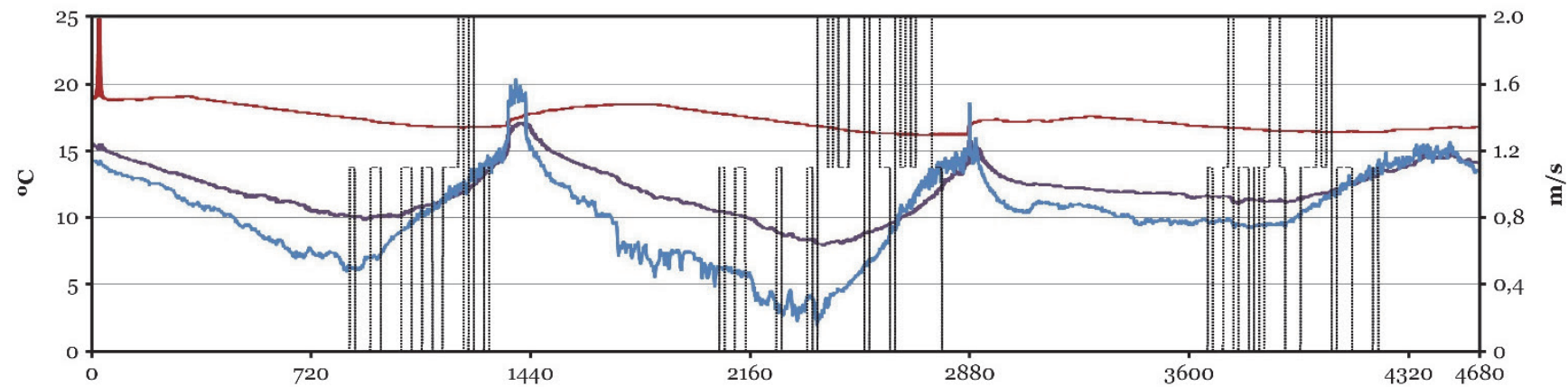

(a)

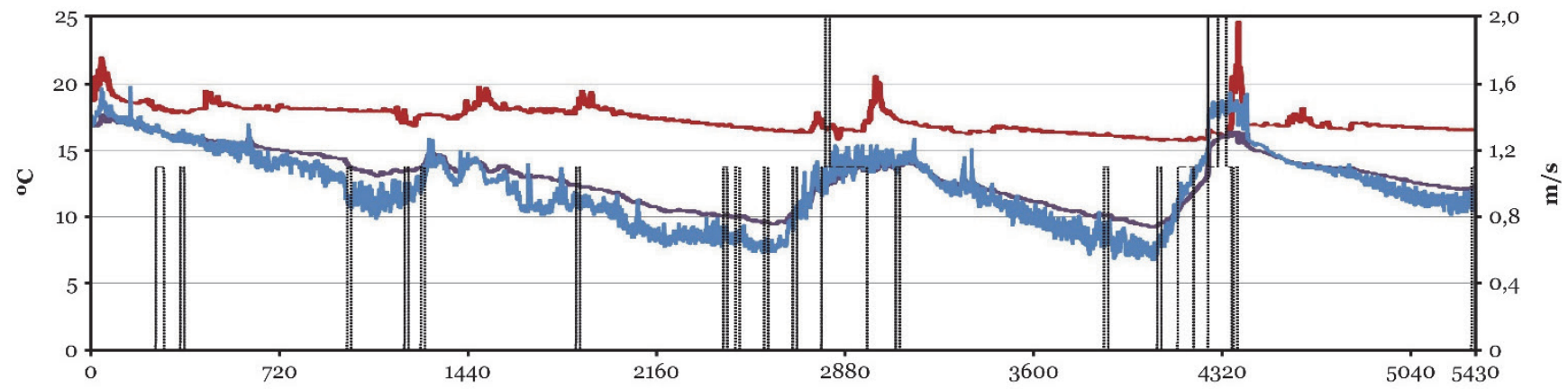

(b)

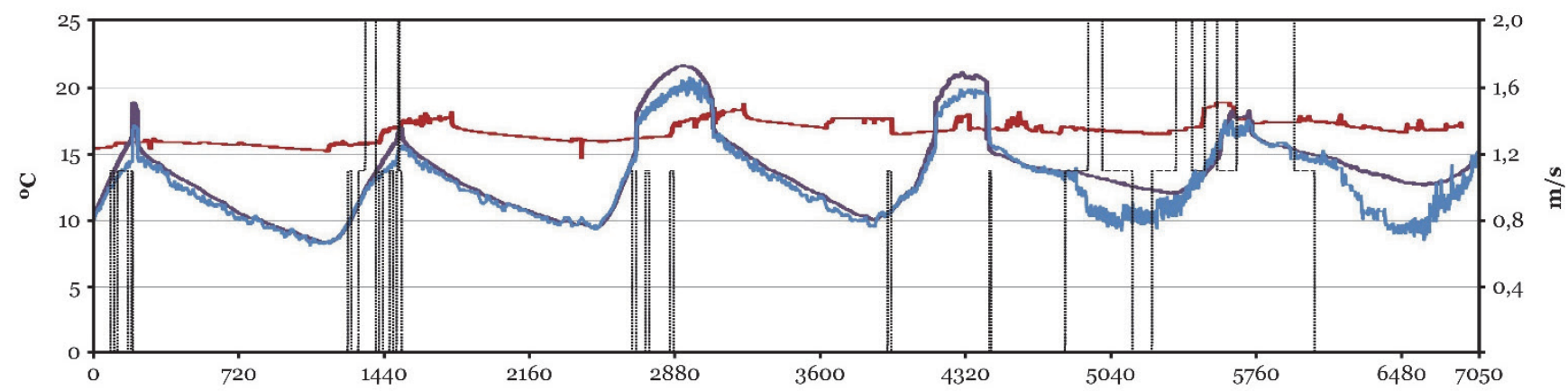

(c)

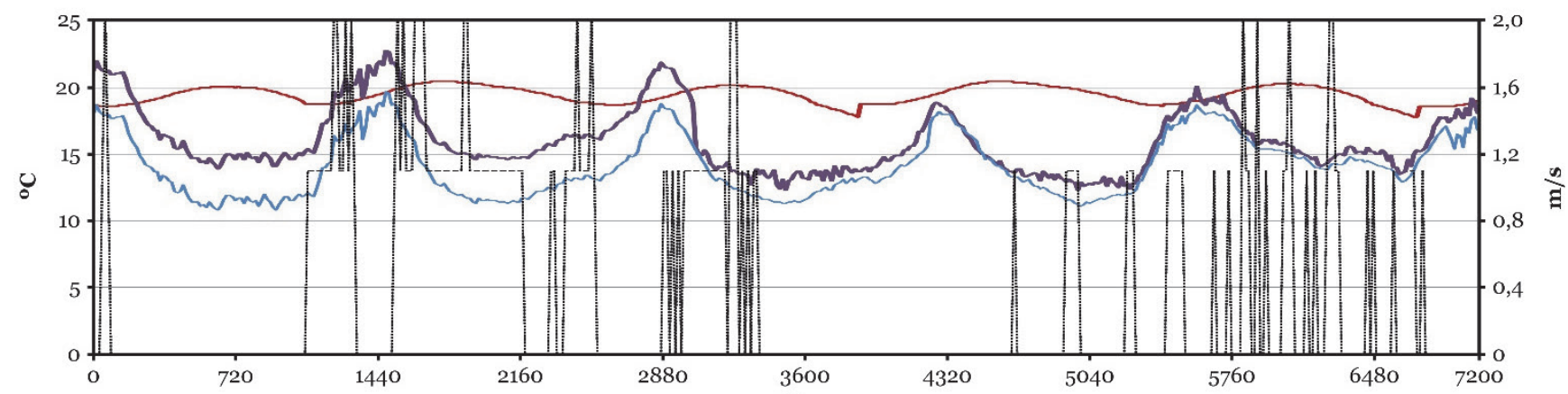

(d)

Figura 3. Datos registrados asociados para las ecuaciones [6] y [7] del análisis cuantitativo mediante termografía infrarroja para los casos de estudio: (a) $\mathrm{C} 1$; (b) $\mathrm{C}_{2}$; (c) $\mathrm{C}_{3}$; (d) $\mathrm{C}_{4}$. En el eje de abscisas se indica el número de instancias de los conjuntos de datos. 
caso de estudio 1. Esta dificultad para obtener un gradiente térmico elevado obligó a descartar los criterios óptimos de realización de los ensayos recomendados por diversos autores (8),(11),(18),(19), en los que se llega incluso a exigir diferencias superiores a $15{ }^{\circ} \mathrm{C}$. Una de las causas que dan lugar a esta circunstancia es atribuida por los autores del presente trabajo a los hábitos de confort propios de la zona, según los cuales no se suelen utilizar sistemas de calefacción, por lo que se asume disconfort térmico en invierno (5). En ocasiones, este diferencial fue negativo al registrar una mayor temperatura en el exterior que en el interior.

Además, es conveniente destacar que no se obtuvieron registros de velocidades inferiores a $1,1 \mathrm{~m} / \mathrm{s}$ a causa de las limitaciones de resolución de la estación meteorológica (Figura 3). Como consecuencia, el valor de viento registrado por el equipo no fue acorde con el real cuando las velocidades no se ajustaron a la resolución de la estación. Por este motivo, los resultados de los métodos que utilizan estas variables (ecuaciones
[6] y [7]), solo serán aptos cuando los valores de velocidad de viento obtenidos sean muy próximos a los reales. Para ello, se comparan los valores de AEMET con los medidos y se utilizan aquellos que presentan una desviación inferior al $10 \%$.

\subsection{Comparación entre diferentes métodos}

Tras analizar los cerramientos objeto de estudio, se obtuvieron los valores de transmitancia térmica según los distintos métodos propuestos (Figura 4), así como la diferencia porcentual entre los valores teóricos (promedio e incertidumbres) y los experimentales (Tablas 7-8).

Se puede apreciar cómo, en los tres primeros casos de estudio, los resultados obtenidos a través de la ISO 9869-1 fueron atípicos (Figura 4) con desviaciones superiores al 30\% debido a que no se colocó un material con una alta conductividad térmica entre el cerramiento y el termoflujómetro. En el cerramiento $\mathrm{C}_{4}$, la disposición de un elemento de estas carac-

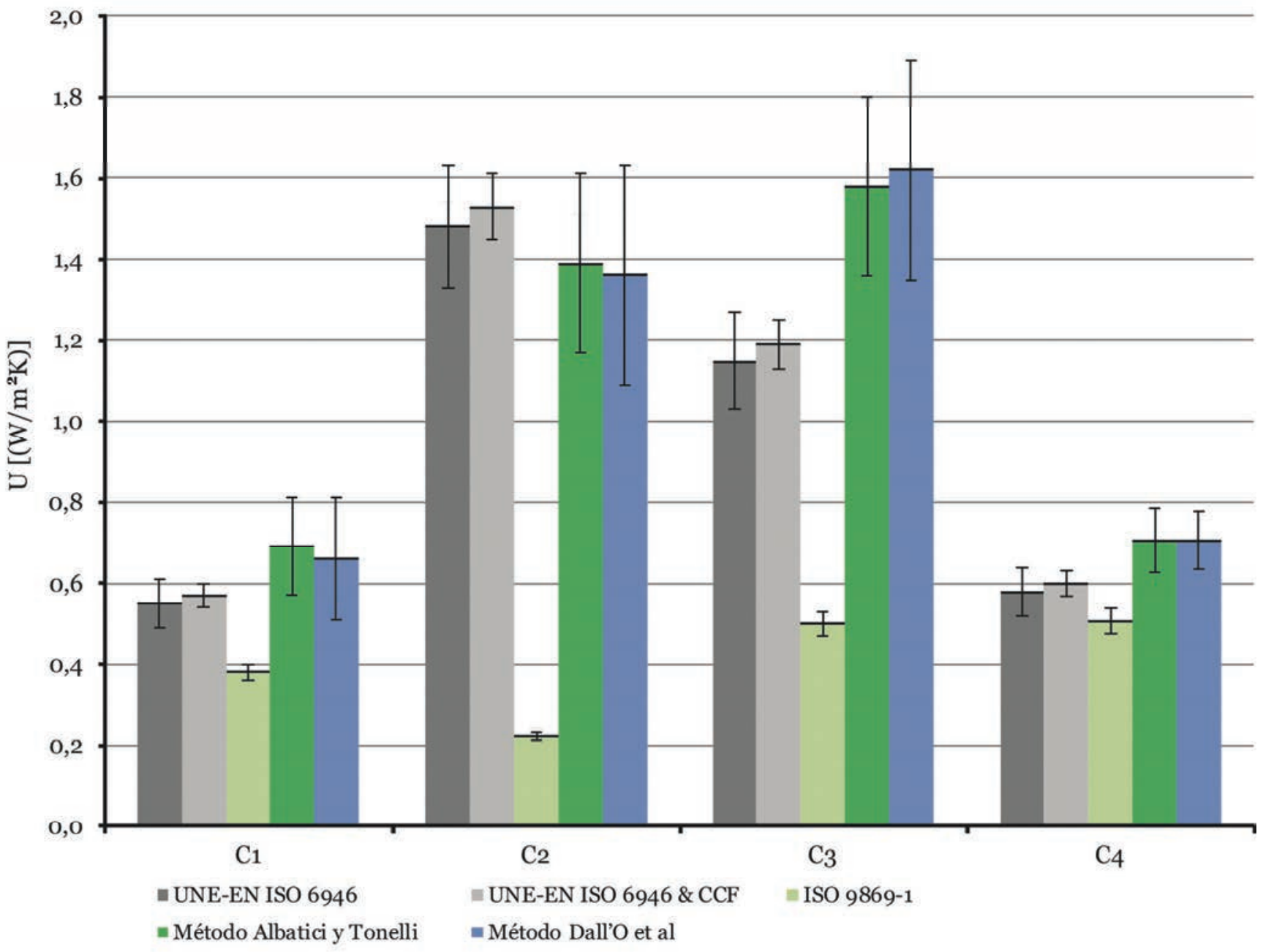

\begin{tabular}{|c|c|c|c|c|c|}
\hline & $\begin{array}{c}\text { UNE-EN ISO 6946 } \\
\text { Ec. [1-3] }\end{array}$ & $\begin{array}{c}\text { UNE-EN ISO 6946 } \\
\text { \& CCF } \\
\text { Ec. [1-4] }\end{array}$ & $\begin{array}{c}\text { ISO 9869-1 } \\
\text { Ec. [5] }\end{array}$ & $\begin{array}{c}\text { Método } \\
\text { Albatici y Tonelli } \\
\text { Ec. [6] }\end{array}$ & $\begin{array}{c}\text { Método } \\
\text { Dall'O et al. } \\
\text { Ec. [7] }\end{array}$ \\
\hline C1 & $0,55 \pm 0,06$ & $0,57 \pm 0,03$ & $0,38 \pm 0,02$ & $0,69 \pm 0,12$ & $0,66 \pm 0,15$ \\
\hline C2 & $1,48 \pm 0,15$ & $1,53 \pm 0,08$ & $0,22 \pm 0,01$ & $1,39 \pm 0,22$ & $1,36 \pm 0,27)$ \\
\hline C3 & $1,15 \pm 0,12$ & $1,19 \pm 0,06$ & $0,50 \pm 0,03$ & $1,58 \pm 0,22$ & $1,62 \pm 0,27)$ \\
\hline C4 & $0,58 \pm 0,06$ & $0,60 \pm 0,03$ & $0,51 \pm 0,03$ & $0,71 \pm 0,08$ & $0,71 \pm 0,07$ \\
\hline
\end{tabular}

Figura 4. Comparación de los resultados obtenidos para los diferentes métodos en los casos de estudio analizados. 
Tabla 7. Relación porcentual entre valores teóricos y experimentales.

\begin{tabular}{|c|c|c|c|c|c|c|c|c|c|}
\hline & \multicolumn{9}{|c|}{ UNE-EN ISO 6946} \\
\hline & \multicolumn{3}{|c|}{$\begin{array}{l}\text { ISO 9869-1 } \\
\text { Ec. }[5]\end{array}$} & \multicolumn{3}{|c|}{$\begin{array}{l}\text { Método Albatici y Tonelli } \\
\text { Ec. [6] }\end{array}$} & \multicolumn{3}{|c|}{$\begin{array}{l}\text { Método Dall'O et al. } \\
\text { Ec. [7] }\end{array}$} \\
\hline & Mín. & Medio & Máx. & Mín. & Medio & Máx. & Mín. & Medio & Máx. \\
\hline $\mathrm{C} 1$ & $-23 \%$ & $-31 \%$ & $-38 \%$ & $41 \%$ & $26 \%$ & $14 \%$ & $35 \%$ & $20 \%$ & $9 \%$ \\
\hline $\mathrm{C} 2$ & $-84 \%$ & $-86 \%$ & $-87 \%$ & $5 \%$ & $-7 \%$ & $-15 \%$ & $3 \%$ & $-9 \%$ & $-17 \%$ \\
\hline $\mathrm{C}_{3}$ & $-52 \%$ & $-57 \%$ & $-61 \%$ & $54 \%$ & $38 \%$ & $25 \%$ & $58 \%$ & $41 \%$ & $28 \%$ \\
\hline $\mathrm{C}_{4}$ & $-2 \%$ & $-13 \%$ & $-21 \%$ & $37 \%$ & $23 \%$ & $11 \%$ & $37 \%$ & $23 \%$ & $11 \%$ \\
\hline
\end{tabular}

Tabla 8. Relación porcentual entre valores teóricos con CCF y experimentales.

\begin{tabular}{|c|c|c|c|c|c|c|c|c|c|}
\hline & \multicolumn{9}{|c|}{ UNE-EN ISO 6946 - CCF } \\
\hline & \multicolumn{3}{|c|}{$\begin{array}{l}\text { ISO 9869-1 } \\
\text { Ec. [5] }\end{array}$} & \multicolumn{3}{|c|}{$\begin{array}{l}\text { Método Albatici y Tonelli } \\
\text { Ec. [6] }\end{array}$} & \multicolumn{3}{|c|}{$\begin{array}{l}\text { Método Dall'O et al. } \\
\text { Ec. [7] }\end{array}$} \\
\hline & Mín. & Medio & Máx. & Mín. & Medio & Máx. & Mín. & Medio & Máx. \\
\hline $\mathrm{C} 1$ & $-30 \%$ & $-34 \%$ & $-37 \%$ & $28 \%$ & $22 \%$ & $15 \%$ & $23 \%$ & $16 \%$ & $10 \%$ \\
\hline $\mathrm{C} 2$ & $-85 \%$ & $-86 \%$ & $-87 \%$ & $-5 \%$ & $-10 \%$ & $-14 \%$ & $-7 \%$ & $-12 \%$ & $-16 \%$ \\
\hline $\mathrm{C}_{3}$ & $-56 \%$ & $-58 \%$ & $-60 \%$ & $40 \%$ & $33 \%$ & $27 \%$ & $44 \%$ & $37 \%$ & $30 \%$ \\
\hline $\mathrm{C}_{4}$ & $-11 \%$ & $-15 \%$ & $-20 \%$ & $25 \%$ & $19 \%$ & $13 \%$ & $25 \%$ & $19 \%$ & $13 \%$ \\
\hline
\end{tabular}

terísticas, así como la prolongación del ensayo a 120 horas, permitió obtener un resultado representativo para el método con una diferencia que osciló entre $13-15 \%$ con respecto al valor de la UNE-EN ISO 6946 (Tablas 7-8). Además, es conveniente destacar que el porcentaje de incertidumbre relativa asociado al resultado $(5,88 \%)$ estuvo dentro de los límites aceptables.

Como se observa en la Figura 4, en el caso de los métodos cuantitativos mediante termografía infrarroja, los valores tuvieron una diferencia inferior al 20\% con respecto a los dos valores teóricos para los cerramientos $\mathrm{C}_{2}$ y C4 (Tablas 7-8). Los resultados obtenidos para ambas ecuaciones fueron muy similares, llegándose a obtener el mismo resultado para el último de los casos de estudio. Sin embargo, los niveles de incertidumbre asociados a los resultados en todos los casos fueron inadmisibles, salvo en la fachada $\mathrm{C}_{4}$, ya que se obtuvieron unos porcentajes de incertidumbre relativa inferiores al $12 \%$. Los autores consideran que esto fue debido a las pocas situaciones con un gradiente de temperatura elevado entre el interior y el exterior y a las velocidades de viento registradas, ya que la resolución de la estación meteorológica no permitió medir con precisión la velocidad del viento. Asimismo, las limitaciones operacionales que implica la utilización de una estación meteorológica, con dificultades para colocarla a escasos centímetros de la pared, provoca que se deba descartar su utilización en favor de una sonda anemométrica, que sí puede colocarse de esta manera.

De los dos métodos teóricos, los valores de transmitancia térmica modificados con el coeficiente propuesto por PérezBella et al. (17) fueron ligeramente superiores a los valores calculados de manera convencional. Se puede apreciar cómo los porcentajes de desviación entre los valores teóricos y los experimentales disminuyeron cuando se aplicó dicha ponderación. Además, los porcentajes estimados de incertidumbre relativa que permiten obtener los valores límite entre los que puede oscilar el valor teórico, permitieron considerar como válidos para el caso $\mathrm{C} 1$ los resultados de los métodos de $\mathrm{Al}-$ batici y Tonelli (8),(9) y Dall'O et al. (10). Esto pone de manifiesto la influencia significativa que puede tener la determinación de la composición del cerramiento y los valores reales de conductividad térmica, ya que pueden variar en función del envejecimiento de los materiales o de las condiciones ambientales, entre otras causas.

El post-procesado de los datos permitió obtener resultados más ajustados que los obtenidos por los diferentes métodos para los datos sin filtrado. Como bien se ha comentado anteriormente, únicamente se consideraron los registros con un diferencial de temperatura superior a $7^{\circ} \mathrm{C}$. La no consideración del filtrado puede provocar errores bastantes significativos en los métodos cuantitativos mediante termografía infrarroja, ya que se llegaron a alcanzar desviaciones superiores al $150 \%$, mientras que para el método termoflujométrico se obtuvo una desviación del 30\% en el valor de transmitancia térmica obtenido en $\mathrm{C}_{4}$. También es conveniente destacar que las condiciones ambientales propias del clima mediterráneo dificultaron la obtención de un número elevado de medidas estables en el tiempo con un gradiente térmico elevado, provocando que el subconjunto de datos obtenido disponga de pocas instancias y los resultados no sean representativos, tal y como ha ocurrido en $\mathrm{C}_{3}$.

Se puede apreciar cómo las condiciones ambientales propias del clima mediterráneo en la estación invernal no son las más propicias para obtener resultados representativos, aunque es posible la obtención de resultados representativos de los diferentes métodos cuando se den unas condiciones ambientales adecuadas. Además, otros aspectos de la realización de los ensayos como la tipología y la ubicación del equipo de medición del viento, así como el material que haga de contacto entre el termoflujómetro y el cerramiento, son aspectos con una gran influencia sobre los resultados.

\section{CONCLUSIONES}

En este trabajo se realiza un estudio comparativo de los métodos de obtención de transmitancia térmica sobre cuatro tipologías de cerramiento opaco en climatología mediterránea en la estación de invierno. La importancia de este trabajo es que pocos estudios han desarrollado análisis cuantitativos y cualitativos de manera global, así como estudios de componentes de la envolvente en los que se utilicen los métodos de evaluación de transmitancia térmica que existen en la actualidad en climas mediterráneos. 
Con base en los resultados obtenidos a raíz de la campaña experimental, se pueden concluir los siguientes puntos:

- Los valores de temperatura propios del clima mediterráneo en invierno, así como las pautas de comportamiento y uso de las viviendas de la zona (caracterizadas por el escaso uso de sistemas de calefacción), dificultan la obtención de registros con una diferencia de temperatura entre el interior y el exterior de los edificios superior a $10{ }^{\circ} \mathrm{C}$, llegando incluso a obtener, en determinados momentos, registros con un gradiente térmico negativo al tener una mayor temperatura en el exterior que en el interior. En cualquier caso, en la campaña experimental se obtuvieron resultados representativos para los diferentes métodos, por lo que resulta necesario asegurar unas condiciones ambientales adecuadas cuando se realicen los ensayos.

- La influencia de la configuración del ensayo es muy significativa en los resultados. La interposición de una pasta de elevada conductividad térmica entre el cerramiento y el termoflujómetro es indispensable para conseguir un adecuado contacto entre los dos y, de esta manera, obtener lecturas correctas de flujo de calor. Asimismo, para la aplicación correcta de los métodos cuantitativos de termografía infrarroja se deben utilizar sondas específicas de altas prestaciones para la medición de la velocidad del viento y descartar, por tanto, el uso de estaciones meteorológicas portátiles debido a las limitaciones que presentaron para la medición de la velocidad de viento local.

- De entre los métodos experimentales, los cuantitativos de termografía infrarroja son los que presentan un comportamiento más aceptable en los diferentes casos de estudio, con valores muy similares para las dos ecuaciones analizadas y desviaciones inferiores al 20\% con respecto al valor teórico, salvo en $\mathrm{C}_{3}$. Sin embargo, para el método termoflujométrico se obtuvieron resultados válidos para el último de los casos de estudio con desviaciones inferiores al 15\%. Así pues, la utilización del método termoflujométrico no se debe descartar en la región climática mediterránea siempre que se den unas condiciones operativas y metrológicas adecuadas y la duración del ensayo se prolongue a más de las 72 h mínimas que exige la ISO 9869-1.

- La utilización de un filtrado de datos para aquellas medidas con un gradiente térmico superior a $7^{\circ} \mathrm{C}$ permitió obtener resultados válidos para algunos de los casos de estudio. Sin embargo, las oscilaciones térmicas propias de la región climática provocan dificultades como, por ejemplo, la obtención de un número elevado de registros con dicho diferencial y estables a lo largo del tiempo, tal y como ocurrió en el caso de estudio 3 .

- El método de cálculo teórico modificado con la corrección propuesta por Pérez-Bella et al. (17) corrige el valor de la UNE-EN ISO 6946 y lo adapta a las condiciones ambientales de la zona. En este sentido, se detecta cómo en aquellos casos en los que se conoce con exactitud la composición del cerramiento, el porcentaje de desviación entre los métodos teóricos y experimentales disminuye con respecto al valor no corregido.

Futuros pasos de esta investigación irán dirigidos a analizar el comportamiento de los métodos experimentales en condiciones más desfavorables como pueden ser las del resto de las estaciones del clima mediterráneo, así como la incorporación de elementos de climatización durante la realización de los ensayos.

\section{AGRADECIMIENTOS}

Los autores agradecen a los revisores sus comentarios y correcciones, ya que mejoraron significativamente el potencial de este documento.

\section{REFERENCIAS}

(1) Li, F. G. N. et al. (2014). Solid-wall U-values: heat flux measurements compared with standard assumptions. Building Research \& Information, 43(2) 238-252. http://dx.doi.org/10.1080/09613218.2014.967977

(2) Stevens, G. \& Bradford, J. (2013). Do U-value insulation? England's field trial of solid wall insulation. eceee 2013 Summer Study, 1269-1280.

(3) Kurtz, F., Monzón, M. \& López-Mesa, B. (2015). Energy and acoustics related obsolescence of social housing of Spain's post- war in less favoured urban areas. The case of Zaragoza. Informes de la Construcción, 67(Extra-1) mo21. http:// dx.doi.org/10.3989/ic.14.062

(4) Rubel, F. \& Kottek, M. (2010). Observed and projected climate shifts 1901-2100 depicted by world maps of the Köppen-Geiger climate classification. Meteorologische Zeitschrift, 19(2) 135-141. http://dx.doi.org/10.1127/o9412948/2010/0430

(5) Sánchez-García, D., Sánchez-Guevara, C. \& Rubio-Bellido, C. (2016). Sevilla The adaptive approach to thermal comfort in Seville. Anales de Edificación, 2 38-48. http://dx.doi.org/10.20868/ade.2016.3197

(6) AENOR. (2012). UNE-EN ISO 6946:2012. Componentes y elementos para la edificación. Resistencia térmica y transmitancia térmica. Método de cálculo. (ISO 6946:2007).

(7) International Organization for Standardization. (2014). ISO 9869-1:2014 - Thermal insulation - Building elements - In situ measurement of thermal resistance and thermal transmittance. Part 1: Heat flow meter method.

(8) Albatici, R. \& Tonelli, A. M. (2010). Infrared thermovision technique for the assessment of thermal transmittance value of opaque building elements on site. Energy and Buildings, 42(11) 2177-2183. http://dx.doi.org/10.1016/j. enbuild.2010.07.010

(9) Albatici, R., Tonelli, A. M. \& Chiogna, M. (2015). A comprehensive experimental approach for the validation of quantitative infrared thermography in the evaluation of building thermal transmittance. Applied Energy, 141 218-228. http:// dx.doi.org/10.1016/j.apenergy.2014.12.035

(10) Dall'O', G., Sarto, L. \& Panza, A. (2013). Infrared screening of residential buildings for energy audit purposes: Results of a field test. Energies, 6(8) 3859-3878. http://dx.doi.org/10.3390/en6083859

(11) Desogus, G., Mura, S. \& Ricciu, R. (2011). Comparing different approaches to in situ measurement of building components thermal resistance. Energy and Buildings, 43(10) 2613-2620. http://dx.doi.org/10.1016/j.enbuild.2011.05.025 
(12) AENOR. (2012). UNE-EN ISO 10456:2012. Materiales y productos para la edificación. Propiedades higrotérmicas. Valores tabulados de diseño y procedimientos para la determinación de los valores térmicos declarados y de diseño. (ISO 10456:2007).

(13) Instituto Eduardo Torroja de Ciencias de la Construcción. (2010). Catálogo de elementos constructivos del CTE.

(14) Clarke, J. A. \& Yaneske, P. P. (2009). A rational approach to the harmonisation of the thermal properties of building materials. Building and Environment, 44(10) 2046-2055. http://dx.doi.org/10.1016/j.buildenv.2009.02.008

(15) Domínguez-Muñoz, F., Anderson, B., Cejudo-López, J. M. \& Carrillo-Andrés, A. (2010). Uncertainty in the thermal conductivity of insulation materials. Energy Build, 42 2159-2168. http://dx.doi.org/10.1016/j.enbuild.2010.07.006

(16) Prada, A., Cappelletti, F., Baggio, P. \& Gasparella, A. (2014). On the effect of material uncertainties in envelope heat transfer simulations. Energy and Buildings, 71 53-6o. http://dx.doi.org/10.1016/j.enbuild.2013.11.083

(17) Pérez-Bella, J. M., Domínguez-Hernández, J., Cano-Suñén, E., Del Coz-Díaz, J. J. \& Álvarez Rabanal, F. P. (2015). A correction factor to approximate the design thermal conductivity of building materials. Application to Spanish façades. Energy and Buildings, 88 153-164. http://dx.doi.org/10.1016/j.enbuild.2014.12.005

(18) Ficco, G., Iannetta, F., Ianniello, E., D’Ambrosio Alfano, F. R. \& Dell'Isola, M. (2015). U-value in situ measurement for energy diagnosis of existing buildings. Energy and Buildings, 104 108-121. http://dx.doi.org/10.1016/j.enbuild.2015.06.071

(19) Asdrubali, F., D’Alessandro, F., Baldinelli, G. \& Bianchi, F. (2014). Evaluating in situ thermal transmittance of green buildings masonries: A case study. Case Studies in Construction Materials, 1 53-59. http://dx.doi.org/10.1016/j. cscm.2014.04.004

(20) Rasooli, A., Itard, L. \& Ferreira, C. I. (2016). A response factor-based method for the rapid in-situ determination of wall's thermal resistance in existing buildings. Energy and Buildings, 119 51-61. http://dx.doi.org/10.1016/j. enbuild.2016.03.009

(21) Lorencio Pérez, S., Molina García, Á. \& García Legaz-Quesada, I. (Universidad Politécnica de Cartagena. Escuela Técnica Superior de Ingeniería Industrial, 2014). Trabajo fin de máster. Análisis termoflujométrico en cerramientos para climas secos.

(22) Domingo de Miguel, J. (Universidad de Navarra. Escuela Técnica Superior de Arquitectura, 2012). Trabajo fin de máster. Evaluación del comportamiento térmico e higrotérmico de cerramientos de madera.

(23) Nardi, I., Paoletti, D., Ambrosini, D., De Rubeis, T. \& Sfarra, S. (2016). U-value assessment by infrared thermography: A comparison of different calculation methods in a Guarded Hot Box. Energy and Buildings, 122 211-221. http://dx.doi. org/10.1016/j.enbuild.2016.04.017

(24) Watanabe, K. (1965). Architectural Planning Fundamentals.

(25) Biddulph, P. et al. (2014). Inferring the thermal resistance and effective thermal mass of a wall using frequent temperature and heat flux measurements. Energy and Buildings, 78 10-16. http://dx.doi.org/10.1016/j.enbuild.2014.04.004

(26) Gobierno de España. (1979). Real Decreto 2429/79, de 6 de julio, por el que se aprueba la Norma Básica de la Edificación NBE-CT-79, sobre Condiciones Térmicas en los Edificios.

(27) Gobierno de España. (2006). Real Decreto 314/20o6, de 17 de marzo, por el que se aprueba el Código Técnico de la Edificación.

(28) Rico Delgado, F. \& Marín García, D. (Universidad de Sevilla. Escuela Técnica Superior de Ingeniería de Edificación, 2009). Tesis. Modelo de diagnóstico por imágenes en edificación mediante la aplicación de tecnologías avanzadas. 